\title{
Clinical characteristics of triple negative breast cancer in Egyptian women: a hospital-based experience
}

\author{
Nivine Gado, Dina Ibrahim, Doaa Atef, Ahmed Kanaan
}

Department of Clinical Oncology, Ain-Shams University, Cairo, Egypt

Received December 29, 2015; Revised May 26, 2016; Accepted May 29, 2016; Published Online June 30, 2016

\section{Original Article}

\begin{abstract}
Purpose: Triple negative breast cancer (TNBC) is an aggressive subtype of breast cancer with poor prognosis despite the high rates of response to chemotherapy. We aim to study the clinical features, factors influencing recurrence and survival outcomes of TNBC patients. Methods: We retrospectively studied the charts of patients with biopsy proven TNBC treated at The Clinical Oncology Department Ain-Shams University between 2009 and 2012. Results: One hundred and forty five patients fulfilled the eligibility criteria. The incidence of TNBC was $10.5 \%$ $15 \%$ with a mean of $12 \%$ of all breast cancer patients. The follow-up duration ranged from six months to four years. The age range was 26 to 78 years. Infiltrating ductal carcinoma represented $93.1 \%$ of the pathologic types. $87 \%$ of patients were free of metastases (M0) at presentation. Clinical stages II and III represented 38 and $39.5 \%$ of the patients. $66 \%$ of patients had modified radical mastectomy. Following surgery, $77.5 \%$ of patients received adjuvant chemotherapy while $61 \%$ of the patients had adjuvant radiation therapy. Anthracyclines based chemotherapy was given to $52 \%$ of patients. Disease-free survival (DFS) of the M0 patients at 20 and 30 months was $92 \%$ and $80 \%$ respectively. Relapse occurred in $23 \%$ of M0 patients. After a mean duration of DFS of 15.1 months, the most common sites of metastases for relapsed M0 patients were pulmonary $(44.8 \%)$, bone $(41.4 \%)$, and locoregional (13.8\%). The median overall survival (ORS) of patients was 18 months (1 - 45 months), whereas for the M1 group of patients the median ORS was 9 months (2 - 29 months). Conclusion: The incidence, pathological characteristics, and clinical behavior of TNBC were similar to what is mentioned in the literature. Adding taxanes to the chemotherapy protocols and using postoperative radiotherapy were both associated with a significant increase in the mean period of DFS, while did not significantly affect the ORS.
\end{abstract}

Keywords: Breast cancer, Triple negative, Recurrence, Survival, Hormonal receptors, HER-2.

\section{Introduction}

Breast Cancer is still the most common malignancy in women worldwide, including Egypt (Cancer registry in Egypt). TNBC (Triple negative breast cancer) is frequently identified by conventional immunehistochemical techniques, as these tumors lack staining for the estrogen receptor (ER), progesterone receptor (PR), and the human epidermal growth factor (HER2). ${ }^{1}$

The St. Gallen international expert consensus 2011 proposed a new classification system for breast cancer based on its division into five subgroups, namely; normal-like, basal, luminal A\&B and HER-2 enriched. A Claudin- low subtype is another described molecular subtype referring to tumors showing features of mesenchymal and mammary stem cells. ${ }^{2}$ The criteria to identify subtypes were further recently refined at the 2013 conference, in that moderate or a strong expression of PR and $\mathrm{Ki}$ - 67 level were both recognized as being important to the surrogate definition of a "Luminal A-like" disease. According to these criteria, the subtypes in question have been defined as: Luminal A ER positive, HER2 negative, Ki - 67 low, and PR high; Luminal B (HER2 negative) - ER positive, HER2 negative, and either $\mathrm{Ki}$ - 67 high or PR low; Luminal B-like (HER2 positive) - ER positive, HER2 over expressed or amplified, any Ki - 67, and any PR; HER2

Corresponding author: Dina Ragab Diab Ibrahim; Department of Clinical Oncology, Ain-Shams University, Cairo, Egypt.

Cite this article as: Gado N, Ibrahim D, Atef D, Kanaan A. Clinical characteristics of triple negative breast cancer in Egyptian women: a hospital-based experience. Int J Cancer Ther Oncol. 2016; 4(2):426. D0I: 10.14319/ijcto.42.6 
positive - HER2 over - expressed or amplified, ER and PR absent; and triple negative - ER and PR absent and HER2 negative. ${ }^{3}$

TNBC patients have a higher risk of metastases and poor overall survival because TNBC is found to be correlated with mutations of BRCA1 gene, over expression of oncoytogenic kinases such as human epidermal growth factor receptor 2, vascular endothelial growth factor - A, insulin-like growth factor - 1 (IGF - 1) / IGF receptor and transforming growth factor - B1.4 These molecular features may have implications for chemotherapy sensitivity to platinum and other directly DNA damaging agents. Using gene expression analysis, Lehmann et al identified six TNBC subtypes namely; basal - like (BL1 \& BL20), an immunomodulatory (IM), a mesenchymal (M), a mesenchymal stem - like (MSL) and a luminal androgen receptor (LAR) subtype. ${ }^{5}$

TNBC is a clinically challenging subtype which accounts for about $9-21 \%$ of all breast cancers, including patients with stages, I - IV. ${ }^{6}$ Compared to other types of breast cancer, TNBC is associated with poor prognosis and overall survival.1, 7, 8 TNBC patients tend to be of younger age less than 50 years. ${ }^{1}{ }^{9}$ TNBC tumors have a shorter median time to relapse and death. TNBC is often locally advanced and of high grade. ${ }^{1}$ The patients have increased risk of local recurrence and metastases, mainly in the lung, brain and soft tissue.10,11,7,8 TNBC is more chemosensitive, and have higher rates of pathological complete remission following neoadjuvant chemotherapy than in other breast cancer types. ${ }^{8}$ The adjuvant chemotherapy is usually recommended in TNBC and should include anthracyclines, taxanes and an alkylating agent. ${ }^{12}$ On the other hand TNBC lacks targeted therapies, and patients do not benefit from anti - estrogen hormonal therapy or trastuzumab. ${ }^{1}$

The aim of this retrospective analysis is to study the clinical characteristics, the prognostic factors of recurrence, disease free survival and overall survival in Egyptian TNBC patients treated at the Department of Clinical Oncology, Ain-Shams University.

\section{Methods and Materials}

We analyzed the institutional medical records, and identified patients who were histopathologically diagnosed with triple negative breast cancer and underwent primary treatment at The Clinical Oncology Department Ain - Shams University between 2009 and 2012. After obtaining approval from the Institutional Review Board at Ain - Shams University Hospitals, a retrospective chart review of patients' demographics, clinical and pathological data was performed. Treatment, follow-up and survival data were obtained from the patients' records. One hundred and forty five patients fulfilled the eligibility criteria which are 18 years or older, immunohistochemistry (IHC) negative expression of ER and PR, and negative HER - 2 neu expression or HER - 2 neu $1+$ or $2+$ expression on IHC accompanied by a negative fluorescence in situ hybridization (FISH) result.

\subsection{Statistical analysis}

Data were revised, coded, tabulated and analyzed using the Statistical Software Package for the Social Sciences, for Windows version 18.0 (SPSS / PASW, Inc., 2009, Chicago, IL). Descriptive statistics (mean and standard deviation) were used to evaluate the DFS of the non metastatic group of patients, and to evaluate the ORS of both metastatic and non-metastatic groups. Chi-square tests were used to assess differences between the DFS and ORS using categorical variables. The overall survival time was calculated in months from the date of diagnosis based on breast biopsy to the date of death or last follow-up. The DFS in months was calculated from the end of the primary treatment until the last date the patient survived without symptoms or signs. Univariate Kaplan - Meier survival curves were plotted, and the log - rank test was used to determine if differences were statistically significant. Statistical significance was defined as alpha less than 0.05 .

\section{Results}

We studied 145 out of the 154 patients diagnosed with TNBC who presented to our department over four years period where 9 patients had incomplete data in their charts (Table 1). The mean age at diagnosis was 52 years. A positive family history was detected in (7.6\%) 11 / 145 of TNBC patients (Table 2).

The most common histopathologic type was infiltrating ductal carcinoma (93.1\%) 135 / 145. Most of the tumors were grade II, 73.7\% (107 / 145). More than half of the tumors were T2 lesions 52.4\% (76 / 145). 96 out of 145 patients had positive lymph nodes (N1, N2, and N3) at diagnosis (66.2\%). Majority of patients were diagnosed with stage II and III breast cancer [38\% (55 / 145) and $40 \%$ (58 / 145) respectively] (Table 3). Most of the patients had modified radical mastectomy (76\%) 110 / 145 , and the rate of breast conservative surgery was only $11.7 \%$ (17 / 145) patients because many patients had stage III cancer (Table 4). Adjuvant chemotherapy was administered in $77.5 \%$ (112 / 145) of patients, of whom 25 patients $22.3 \%$ (25 / 112) developed recurrence. 14 patients out of 145 (9.7\%) received neoadjuvant chemotherapy, with recurrence in 4 patients (28.6\%). 89 of 145 patients (61.3\%) received adjuvant radiotherapy, followed by recurrence in only 19 / 89 patients (21.3\%). Anthracyclines - based regimens were used as adjuvant or neoadjuvant in (52.4\%) 76 / 145 of patients. 
Table1: Summary of breast cancer registration in AinShams Oncology Department from 2009 to 2012

\begin{tabular}{ccccc}
\hline \hline Year & $\begin{array}{c}\text { No. of all } \\
\text { cancer } \\
\text { patients }\end{array}$ & $\begin{array}{c}\text { No. of } \\
\text { breast } \\
\text { cancer } \\
\text { patients }\end{array}$ & $\begin{array}{c}\text { No. of } \\
\text { TNBC } \\
\text { patients }\end{array}$ & $\begin{array}{c}\text { \% of TNBC } \\
\text { of all breast } \\
\text { cancer } \\
\text { patients }\end{array}$ \\
\hline 2009 & 1441 & 315 & 47 & $15 \%$ \\
2010 & 1536 & 333 & 40 & $12 \%$ \\
2011 & 1563 & 294 & 31 & $10.5 \%$ \\
2012 & 1489 & 324 & 36 & $11 \%$ \\
\hline Total & 6029 & 1266 & 154 & $12 \%$ \\
\hline \hline
\end{tabular}

Table 2: Patients characteristics $(n=145)$

\begin{tabular}{ccc}
\hline \hline Variable & No. of patients & $\%$ \\
\hline Age at initial diagnosis, years & 52 & \\
Mean & $27-78$ & \\
Range & & \\
& & \\
Menopausal history & 70 & 48.3 \\
Premenopausal & 70 & 48.3 \\
Postmenopausal & 5 & 3.4 \\
Perimenopausal & & \\
Family History & 11 & 7.6 \\
Positive & 128 & 89 \\
Negative & 6 & 4 \\
Unavailable &
\end{tabular}

The DFS for the M0 patients (126) at 20 and 30 months was about $92 \%$ and $80 \%$ respectively (Figure 1). After a mean period of disease - free survival (DFS) of 15.1 months, 29 patients out of 126 (23\%) relapsed. The most common sites of relapse were the lung (13 patients, $44.8 \%)$, bone (12 patients, 41.4\%), and locoregional (4 patients, 13.8\%) (Table 8). The overall survival of both M0 and M1 patients at 20 months and 30 months was about $98 \%$ and $88 \%$ respectively (Figure $2)$. The median ORS for the M0 group of patients was 18 months (1 - 45 months), whereas for the M1 group of patients the median ORS was 9 months (2 - 29 months) (Table 5).

Except for tumor stage which had direct effect on the recurrence and overall survival $(P<0.05)$, univariate analysis showed that all other risk factors had no statistically significant relationship to either recurrence or overall survival (Tables 6, 7). Patients with stages 0 and I had no recurrence compared to patients with advanced stages. Similarly, the overall survival of patients with early stages was better than with advanced stages as shown in Table (5). The disease stage, the type of chemotherapy and the adjuvant radiation therapy had direct statistically significant effect on the DFS $(P<0.05)$ (Table 6).
Table 3: Tumor characteristics ( $\mathrm{n}=145$ patients)

\begin{tabular}{|c|c|c|}
\hline Variable & No of patients & $\%$ \\
\hline \multicolumn{3}{|l|}{ Histologic type } \\
\hline Infiltrating ductal carcinoma & 135 & 93.1 \\
\hline Infiltrating lobular & 9 & 6.2 \\
\hline carcinoma & 1 & 0.7 \\
\hline \multicolumn{3}{|l|}{ Ductal carcinoma in situ } \\
\hline \multicolumn{3}{|l|}{ Histologic grade } \\
\hline I & 4 & 2.7 \\
\hline II & 107 & 73.7 \\
\hline III & 23 & 16 \\
\hline Unavailable & 11 & 7.6 \\
\hline \multicolumn{3}{|l|}{ Tumor size } \\
\hline Tis & 1 & 0.7 \\
\hline $\mathrm{T} 1$ & 14 & 9.7 \\
\hline $\mathrm{T} 2$ & 76 & 52.4 \\
\hline T3 & 38 & 26.2 \\
\hline $\mathrm{T} 4$ & 9 & 6.2 \\
\hline Unavailable & 7 & 4.8 \\
\hline \multicolumn{3}{|l|}{ Nodal involvement } \\
\hline N0 & 42 & 29 \\
\hline N1 & 40 & 27.5 \\
\hline N2 & 34 & 23.5 \\
\hline N3 & 22 & 15 \\
\hline Unavailable & 7 & 5 \\
\hline \multicolumn{3}{|l|}{ Staging } \\
\hline Stage 0 & 1 & 0.7 \\
\hline Stage 1 & 9 & 6.3 \\
\hline Stage 2 & 55 & 38 \\
\hline Stage 3 & 58 & 40 \\
\hline Stage 4 & 19 & 13 \\
\hline Unavailable & 3 & 2 \\
\hline \multicolumn{3}{|l|}{ Metastasis at diagnosis } \\
\hline M0 & 126 & 87 \\
\hline M1 & 19 & 13 \\
\hline
\end{tabular}

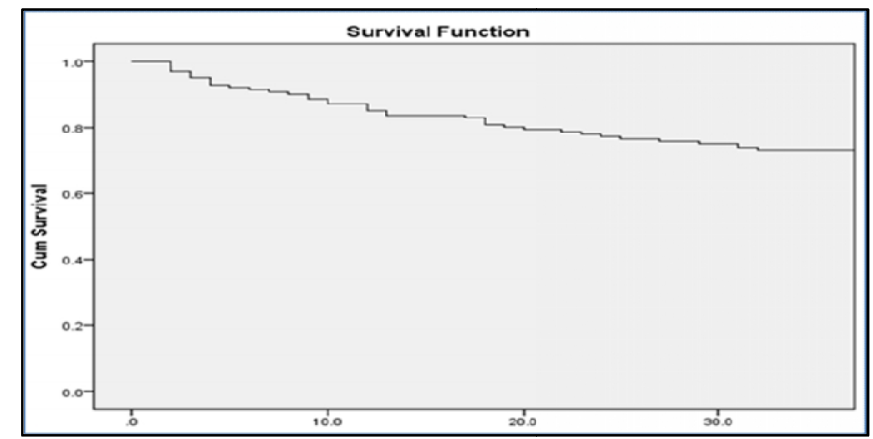

Figure 1: Disease Free Survival of M0 triple negative breast cancer patients at Department of Clinical Oncology, Ain Shams University (2009-2012). 


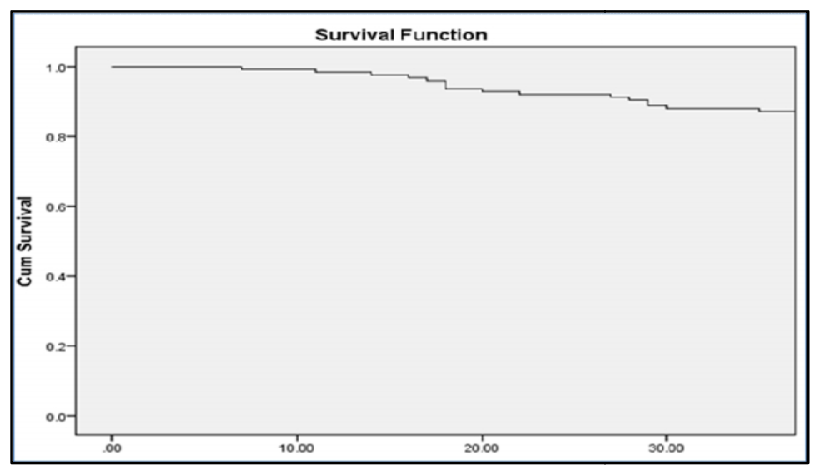

Figure 2: Overall survival of both M1 and M0 triple negative breast cancer patients at Department of Clinical Oncology, Ain Shams University (2009-2012).

Table 4: Treatment of M0 and M1 ( $\mathrm{n}=145)$

\begin{tabular}{ccc}
\hline \hline Variable & No. of patients & $\%$ \\
\hline Surgery & & \\
Modified radical mastectomy & 110 & 76 \\
Conservative & 17 & 11.7 \\
Simple mastectomy & 7 & 4.8 \\
Only biopsy & 11 & 7.5 \\
Chemotherapy & & \\
Adjuvant & 112 & 77.2 \\
Neoadjuvant & 14 & 9.7 \\
Palliative & 16 & 11 \\
Unavailable & 3 & 2.1 \\
The chemotherapy regimen & & \\
Anthracyclines based & & \\
Sequential & 76 & 52.4 \\
Anthracyclines/Taxanes & 60 & 41.4 \\
Other regimens & 6 & 4.1 \\
Unavailable & 3 & 2.1 \\
& & \\
Radiotherapy & & \\
Adjuvant & 89 & 61.3 \\
Palliative & 10 & 71.7 \\
Not given & 46 & \\
& & \\
\hline \hline
\end{tabular}

Table 5: Treatment outcome

\begin{tabular}{ccc}
\hline \hline Variable & No. of patients & $\%$ \\
\hline $\begin{array}{c}\text { Disease free survival for M0 } \\
\text { patients (n =126) }\end{array}$ & & \\
$\quad$ No recurrence & 97 & 77 \\
$\quad$ Recurrence & 29 & 23 \\
$\quad \begin{array}{l}\text { Progression free survival for M1 } \\
\text { patients (n = 19) }\end{array}$ & 7 & 36.8 \\
$\quad \begin{array}{l}\text { Progression or new } \\
\quad \text { metastatic site }\end{array}$ & 12 & 63.2 \\
$\quad \begin{array}{l}\text { No Progression or new } \\
\quad \text { metastatic site }\end{array}$ & 115 & 91.26 \\
$\begin{array}{l}\text { Overall survival for M0 (n =126) } \\
\text { Overall survival for M1 (n =19) }\end{array}$ & 13 & 68.4 \\
\hline \hline
\end{tabular}

\section{Discussion}

The current study is a retrospective review of 145 patients with TNBC in our institution over a 4-year period (2009 - 2012). It is one of the few studies of TNBC in non - Western countries. In our study, the rate of TNBC was $12 \%$, which is comparable to other studies (9 - 21\%) in non - Western countries ${ }^{\mathbf{1 0}}, \mathbf{1 3}, \mathbf{1 4}$, and in Western countries. ${ }^{15}$, 11,6,16 Triple - negative breast tumors have been characterized by several aggressive clinicopathologic features including onset at younger age, higher mean tumor size, higher-grade tumors, and, in some cases, a higher rate of node positivity. ${ }^{1,17}$

The mean age at diagnosis in this study is 52 years, similar to the mean age at presentation documented by many studies. ${ }^{\mathbf{1 8 , 1 9 , 1 , 2 0}}$ Some studies showed different mean ages of TNBC patients $44-45$ years in Korea and Turkey ${ }^{21,22}$, and 56 years in Japan. ${ }^{23}$

Ghosn et al $^{\mathbf{1 0}}$ and Fakhoury ${ }^{\mathbf{2 4}}$ reported a positive family history in $10 \%$ of patients with TNBC in Lebanon compared with $1 \%$ of patients with breast cancer when all phenotypes are included. In our study, a positive family history was documented in $7 \%$ of patients. Similar incidence of less than $10 \%$ was found by Rais et al. ${ }^{13}$ Higher incidence of positive family history was documented by other studies, both Kwan et al, and Fayaz et al $20,2520 \%$ and 28\% in Phipps et al. ${ }^{26}$

The histological characteristics in this current study showed that invasive ductal carcinoma represented 93.3\% in accordance with $90 \%$ in many studies. ${ }^{27-32}$ Invasive ductal carcinoma was most predominant in Singapore and Japan (93\% and 95\%, respectively). ${ }^{23,33}$ Invasive lobular carcinoma was interestingly reported in $2 \%$ of TNBC patients in Singapore ${ }^{23}, 4 \%$ in Kuwait ${ }^{25}$, $2.3 \%$ in Italy ${ }^{34}$ vs. $6 \%$ in our study. This may represent the pleomorphic subtype of lobular carcinoma. ${ }^{35}$ Triple negative breast cancers are mainly high-grade tumors with high mitotic index and marked cellular proliferation.1, 21, 28, 36-38 Most studies reported predominance of high histologic grade that ranged from $55-62 \%$. Contrary in our study, grade II tumors represented $74 \%$, while grade III was $16 \%$.

The aggressiveness of this type of breast cancer and its highly proliferating nature mean that it also tends to be diagnosed at a later stage. ${ }^{18,39}$ Our analysis found that stages 0 - I represented $45 \%$ while stages III and IV represented $52.5 \%$. This is comparable to some publications, which reported $34 \%$ stage III vs. 15\% stage I in a study by Pogoda et $a l^{40}, 8 \%$ stage I vs. $28 \%$ stage III in a study by Rais et al. ${ }^{13}$ Fayaz et al ${ }^{25}$ reported $56 \%$ of patients with stages, I and II while stage III represented $37 \%$. Stark et al ${ }^{41}$, studied a series of 1236 patients with invasive ductal carcinoma, they concluded that women with stages III and IV were 16 times more likely to have triple - negative tumors than those with early stages 
[odds ratio (OR) 16.4; 95\% confidence interval (CI): 7.8 $-34.2]$.

Triple - negative breast cancers are commonly of large size at presentation. ${ }^{29,36-38,42-45}$ Tumor size was the most relevant prognostic factor for survival in some studies. In a study by Pogoda et al, the hazard ratio of recurrence in patients with a tumor $>5 \mathrm{~cm}$ was 16 times higher than in patients with tumor $>2 \mathrm{~cm}$. The authors also found a higher hazard for death in patients with large tumors (HR $=8.21$ in tumors $>5 \mathrm{~cm}){ }^{40}$ Similarly, Hamm et al reported that the tumor size was the most important prognostic factor. ${ }^{15}$ Moreover, tumors $<2 \mathrm{~cm}$ with negative nodes were documented to have a high recurrence rate. ${ }^{38,45}$ The current study documented predominance of $\mathrm{T} 2$ tumors.

The detection of positive lymph nodes at presentation in TNBC patients is interestingly contradictory in different studies. Dent $e t a l^{1}$ found that the rate of node positivity was slightly higher in node positive TNBC compared with other types of breast cancer (54.6\% versus $45.6 \%$, respectively; $P=0.02$ ). Similar results of positive lymph nodes were reported by Fayaz et al and Ghosn et al(58 and $50 \%$ respectively). ${ }^{25,10}$ Lin et al and related studies demonstrated that TNBC was less likely to be lymph node positive (38 and $41 \%$, respectively). ${ }^{17,7} \mathrm{~A}$ much lower incidence of positive lymph nodes was detected in Japanese patients by Ishikawa et al. $^{33}$ In the current study, positive lymph node was $66 \%$ possibly due to the tendency to late presentation of patients in the social stratum that we treat at our center.

Despite the poor clinical outcome and DFS, the more aggressive clinical course in the metastatic setting, TNBC is sensitive to standard chemotherapy. Chemotherapy is the standard treatment of TNBC in the adjuvant, neoadjuvant, and metastatic settings due to the lack of response to traditional hormonal therapies and targeted therapies. ${ }^{1}$ TNBC patients have a higher pathologic complete response (pCR) than non - TNBC, and also better survival compared to TNBC patients who do not achieve pCR. ${ }^{8}$ The pCR rate was $29 \%$ in patients who received neoadjuvant anthracyclines based chemotherapy and $38 \%$ after anthracyclines and taxanes combined treatment. ${ }^{46,47}$ In another study, two thirds of patients received neoadjuvant anthracycline- taxane chemotherapy and only $15 \%$ of them achieved pCR; recurrence occurred in almost half of patients in this group. ${ }^{40}$ Our study, showed that the majority of M0 patients $(\mathrm{n}=126)$ received adjuvant chemotherapy (89\%) in the form of anthracyclines based regimens (52.3\%). There was insignificant difference between patients who received adjuvant vs. neoadjuvant chemotherapy as regards the recurrence status (DFS was 15.48 months and 11.75 months respectively) and as regards the overall survival, (ORS was $91.07 \%$ and $92.86 \%$, respectively). Similarly the type of chemotherapy, whether combined anthracyclines and taxanes or taxanes alone in M0 patients $(\mathrm{n}=126)$ did not significantly affect both the DFS $(76.67 \%$ and $77.27 \%$ respectively) and the ORS (95\% and $87.88 \%$ respectively).

Triple negative breast cancers have been associated with lower disease - free survival, a higher predisposition to visceral metastases and poorer outcome when compared to other subtypes of breast cancer.18, 1,48,49 Women with triple negative breast cancer typically tend to develop recurrence during the first 3 years after therapy with a rapid decline thereafter; and the majority of deaths occur in the first 5 years post treatment. Patients with non - TNBC have more consistent rates of recurrence. ${ }^{1,8}$ In both studies by Ghosn et al, and Haffty et al10,50, the peak of recurrence occurred after 6 - 18 months. Fayaz et $a 2^{25}$ reported the peak of recurrence at 30 months. We observed the same phenomenon where after a mean period of DFS of 15.1 months, 29 out of 126 metastases - free patients developed recurrence. The lung was the most common site of metastases followed by bone and locoregional recurrence (Table 8). The ORS for the non - metastatic group at presentation of patients was $87 \%$ (11 / 126), whereas for the metastatic group of patients M1 the ORS was 31.6\% (6 / 19). The Mayo Clinic Study reported an overall survival at 5 years after surgery of $85 \%$ vs. $87 \%$ in the present study. In Kaplan et al. ${ }^{6}$ study, the 5 - year disease - free survival and ORS in TNBC patients were $84 \%$ and $81 \%$ respectively. The variable results between studies may be dependent on the difference in staging of breast cancer, in the present study majority of patients were stage II and III, whereas in Kaplan's study about $80 \%$ of patients presented with stages, I and II.

To date, not a single targeted therapy has been approved for the treatment of TNBC, and cytotoxic chemotherapy remains the standard treatment. ${ }^{51}$ Novel candidate compounds for TNBC have entered phase II and phase III trials and will likely require patient stratification before therapy. Examples of these tailored approaches include poly (adenosine diphosphate - ribose) polymerase (PARP) inhibitors for BRCA - mutated TNBC, antiandrogens for androgen receptor (AR) - positive TNBC, fibroblast growth factor receptor (FGFR) inhibitors for TNBC harboring FGFR amplifications, and gamma - secretase inhibitors for TNBC with mutations in the PEST domain of NOTCH proteins. Well - designed clinical trials of molecularly targeted therapy for different subgroups of TNBC are necessary. ${ }^{52}$ 
Table 6: Factors influencing recurrence and mean duration of DFS for M0 disease ( $\mathrm{n}=126)$

\begin{tabular}{|c|c|c|c|c|c|c|}
\hline Variable & Recurrence & $\begin{array}{c}\text { No } \\
\text { recurrence }\end{array}$ & $\begin{array}{c}\text { Total } \\
\text { number }\end{array}$ & $P$ value & $\begin{array}{c}\text { Mean } \\
\text { duration of } \\
\text { DFS } \pm \text { SD }\end{array}$ & $P$ value \\
\hline \multicolumn{7}{|l|}{ Stage } \\
\hline 0 & 0 & 1 & 1 & & & \\
\hline I & $0(0 \%)$ & 9 & 9 & & & \\
\hline II & $6(11 \%)$ & 49 & 55 & & & 0.002 \\
\hline III & $22(38 \%)$ & 36 & 58 & & & \\
\hline \multicolumn{7}{|l|}{ Chemotherapy Type } \\
\hline Anthracyclines/taxanes & $14(23.3 \%)$ & 46 & 60 & 0.9 (NS) & $19.3 \pm 11.7$ & 0.031 \\
\hline Anthracyclines & $15(22.7 \%)$ & 51 & 66 & & & \\
\hline \multicolumn{7}{|l|}{ Radiotherapy } \\
\hline Adjuvant & $19(21.4 \%)$ & 70 & 89 & 0.5 (NS) & $18 \pm 10.4$ & 0.027 \\
\hline No adjuvant & $10(27 \%)$ & 27 & 37 & & & \\
\hline
\end{tabular}

SD: standard deviation

Table 7: Factors influencing overall survival (ORS)of M1 and M0 patients $(n=145)$

\begin{tabular}{ccccc}
\hline \hline & Alive & Dead & OS \% & $P$ value \\
\hline Stage & & & & \\
0 & 1 & 0 & 100 & \\
I & 9 & 0 & 100 & \\
II & 52 & 3 & 95 & 0.03 \\
III & 50 & 8 & 86.2 & \\
IV & 13 & 6 & 68.4 & \\
\hline \hline
\end{tabular}

Table 8: Pattern of recurrence in M0 patients $(n=29 / 126)$

\begin{tabular}{ccc}
\hline \hline Site & Number & $\%$ \\
\hline Lung & 13 & 44.8 \\
Bone & 12 & 41.4 \\
Locoregional & 4 & 13.8 \\
\hline \hline
\end{tabular}

PARP enzymes are critical to cell recovery from DNA damage. When PARP1, the most abundant member of the PARP family, is inhibited, double - strand DNA breaks accumulate and under normal conditions are repaired via the BRCA pathway-dependent homologous recombination mechanism. ${ }^{53}$ The efficacy and safety of PARP inhibitors are tested in clinical trials in both BRCA - mutated and triple negative breast cancers because of the shared clinicopathologic characteristics. ${ }^{54}$-56 In a single arm study olaparib (400 mg orally twice daily) was administered to women with BRCA1 and / or BRCA2 - deficient, advanced breast cancer (of which > 50 percent were triple - negative). Olaparib resulted in an overall response rate of 41 percent and PFS of 5.7 months. The drug was generally well tolerated with the most commonly reported grade 3 adverse events being fatigue, nausea, and vomiting. ${ }^{54,57}$ On the other hand, another study showed that olaparib had no activity in breast cancer outside of patients with known germline BRCA mutations, although epithelial ovarian cancer appears to respond similarly regardless of germline status. ${ }^{58}$

The role of cetuximab; an anti - EFGR monoclonal antibody in patients with metastatic TNBC was studied ${ }^{59}$ by comparing cetuximab plus cisplatin versus cisplatin alone. The authors demonstrated an overall better response rate of $20 \%$ when compared to a $10 \%$ overall better response rate with cisplatin alone. Cisplatin plus cetuximab also resulted in longer progression free survival (PFS) compared with cisplatin alone (median, 3.7 vs. 1.5 months) with a corresponding median OS of 12.9 versus 9.4 months. Common grade 3 / 4 adverse events included acne - like rash, neutropenia, and fatigue. Despite the longer PFS and OS, the trial failed to reach its primary endpoint, which is the overall response rate compared to the single regimen. Additionally cetuximab induced diarrhea was a concern when added to chemotherapy as carboplatin and irinotecan despite an increased overall response rate in the TNBC subset of O'Shaughnessy's phase II trial. ${ }^{60}$ The potential efficacy of anti - EGFR strategies needs further investigation with more trials.

\section{Conclusion}

This study documents the clinical experience of TNBC at our institution. The results of the study accord with the literature data of characteristics of TNBC in terms of young age at presentation, high - grade tumors, late stages at diagnosis, and short disease - free survival. We acknowledge the limitations of this study inherent to most retrospective studies, the relatively small sample 
size, and the lack of BRCA gene mutations' studies due to the limited financial resources. However, our results are unique to Egypt, are one of the very few studies of TNBC in the Middle East, and represent a single - institution approach to patient care.

Tumor stage, chemotherapy type and adjuvant radiation therapy statistically influenced the recurrence and the duration of disease - free survival. In addition, the tumor stage statistically influenced the overall survival of both the metastatic and non - metastatic TNBC in our study.

Unfortunately, TNBC as an aggressive subtype of breast cancer has limited treatment options when it does not respond to or progresses after the standard and /or taxanes based regimens. More research should be directed at identifying molecules and pathways that may be effective targets for new drugs.

\section{Conflict of interest}

The authors declare that they have no conflicts of interest. The authors alone are responsible for the content and writing of the paper.

\section{References}

1. Dent R, Trudeau M, Pritchard KI, et al. Triple negative breast cancer: Clinical features and patterns of recurrence. Clin Cancer Res. 2007; 13: 4429-34.

2. Prat A, Parker JS, Karginova O, et al. Phenotypic and molecular characterization of the claudin-low intrinsic subtype of breast cancer. Breast Cancer Res. 2010; 12(5):R68.

3. Yanagawa M, Ikemot K, Kawauchi S, et al. Luminal A and luminal B (HER2 negative) subtypes of breast cancer consist of a mixture of tumors with different genotype. BMC Res Notes. 2012; 5:376.

4. Jia LYI, Shanmugam MK, Sethi G, et al. Potential role of targeted therapies in the treatment of triple-negative breast cancer. Anticancer drugs. 2016;27(3):147-55. In process.

5. Lehmann BD, Bauer JA, Chen X, et al. Identification of human triple-negative breast cancer subtypes and preclinical models for selection of targeted therapies. J Clin Invest. 2011; 121:2750-67.

6. Kaplan HG, Malmgren JA. Impact of triple negative phenotype on breast cancer prognosis. Breast J. 2008; 14:456-63.

7. Lin NU, Vanderplas A, Hughes ME, et al. Clinicopathologic features, patterns of recurrence, and survival among women with triple-negative breast cancer in the National comprehensive cancer network. Cancer. 2012.
8. Liedke C, Mazouni C, Hess KR, et al. Response to neoadjuvant therapy and long-term survival in patients with triple-negative breast cancer. J Clin Oncol. 2008; 26:1275-81.

9. Rakha EA, El-Sayed M, Green A, et al. Prognostic markers in triple-negative breast cancer. Cancer. 2007; 109:25-32.

10. Ghosn M, Hajj C, Kattan J, et al. Triple-negative breast cancer in Lebanon: a case series. Oncologist. 2011; 16:1552-6.

11. Heitz F, Harter P, Traut A, et al. Cerebral metastases (CM) in breast cancer (BC) with focus on triple-negative tumors. J Clin Oncol. 2008;26 (15 suppl) (abstract 1010).

12. Goldhirsch A, Wood WC, Coates AS, et al. Panel members. Strategies for subtypes-dealing with the diversity of breast cancer: highlights of the St. Gallen International Expert Consensus on the Primary Therapy of Early Breast Cancer. Ann Oncol. 2011;22(8):1736-47.

13. Rais G, Raissouni S, Aitelhaj M, et al. Triple-negative breast cancer in women: clinicopathologic and therapeutic study at the National Institute of Oncology. BMC Women's Health. 2012; 12:35.

14. Abulkair O, Moghraby J, Badri M, et al. Clinicopathologic features and prognosis of triple negative breast cancer in patients 40 years of age and younger in Saudi Arabia. Hematol Oncol Stem Cell Ther. 2012;5(2);101-6.

15. Hamm C, El-Masri M, Poliquin G, et al. A single-center chart review exploring the adjusted association between breast cancer phenotype and prognosis. Curr Oncol. 2011; 18:191-6.

16. Lee JA, Kim KI, Bae JW, et al. Korean Breast Cancer Society. Triple negative breast cancer in Korea-distinct biology with different impact of prognostic factors on survival. Breast Cancer Res Treat. 2010; 123:177-87.

17. Carey L, Perou C, Livasy C, et al. Race, breast cancer subtypes, and survival in the Carolina breast cancer study. JAMA. 2006; 295:2492-502.

18. Bauer KR, Brown M, Cress RD et al. Descriptive analysis of estrogen receptor (ER) negative, progesterone receptor (PR)-negative, and HER2-negative invasive breast cancer, the so-called triple negative phenotype: A population-based study from the California Cancer Registry. Cancer 2007; 109:1721-8.

19. Tawfik O, Davis K, Kimler BF, et al. Clinicopathological characteristics of triple negative invasive mammary carcinomas in African-American versus Caucasian women. Ann Clin lab Sci. 2010 Fall;40(4):315-23.

20. Kwan ML, Kushi LH, Weltzien E, et al. Epidemiology of breast cancer subtypes in two 
prospective cohort studies of breast cancer survivors. Breast cancer Res. 2009; 11(3):R31.

21. Park YH, Lee SJ, Cho EY, et al. Clinical Relevance of TNM staging systems according to breast cancer subtypes. Ann Oncol. 2011;22(7):1554-60.

22. Akosky S, Dizdar O, Harputluoglu H, et al. Demographic, clinical, and pathological characteristics of Turkish triple-negative breast cancer: single center experience. Ann Oncol. 2007;18(11):1904-6.

23. Thike AA, Cheok PY, Jara-Lazaro AR, et al. Triple-negative breast cancer: clinicopathological characteristics and relationship with basal-like breast cancer. Mod Pathol. 2010; 23(1): 123-33.

24. Fakhoury W. Prognostic Factor in Breast Cancer; Correlation with Other Factors; Estrogen and Progesterone Receptors, MIB and Histoprognostic Grade. Thesis. Saint-Joseph University, Beirut, Lebanon, 1996-1997:1-50.

25. Fayaz MS, El-Sherify MS, El-Basmy A et al. Clinicopathological features and prognosis of triple negative breast cancer in Kuwait: A comparative/perspective analysis. Reports of practical oncology and radiotherapy. 2014; 19:173-81.

26. Phipps AI, Chlebowski RT, Prentice R, et al. Body size, physical activity, and risk of triple-negative and estrogen receptor-positive breast cancer. Cancer Epidemiol Biomarkers Prev. 2011;20(3):454-63.

27. Lin C, Chien SY, Chen LS, et al. Triple negative breast carcinoma is a prognostic factor in Taiwanese women. BMC Cancer. 2009; 18; 9:192.

28. Fulford LG, Easton DF, Reis-Filho JS, et al. Specific morphological features predictive for the basal phenotype in grade 3 invasive ductal carcinoma of breast. Histopathology. 2006; 49:22-34.

29. Lakhani SR, Reis-Filho JS, Fulford L, et al. Prediction of BRCA1 status in patients with breast cancer using estrogen receptor and basal phenotype. Clin Cancer Res. 2005;15;11(14):5175-80.

30. Livasy CA, Karaca G, Nanda R, et al. Phenotypic evaluation of the basal-like subtype of invasive breast carcinoma. Mod Pathol. 2006; 19:264-71.

31. Tsuda H, Takarabe T, Hasegawa T, et al. Myoepithelial differentiation in high grade invasive ductal carcinomas with large central acellular zones. Hum Pathol. 1999; 30:1134-9.

32. Tsuda H, Takarabe $\mathrm{T}$, Hasegawa $\mathrm{F}$, et al. Large, central acellular zones indicating myoepithelial tumor differentiation in high-grade invasive ductal carcinomas as markers of predisposition to lung and brain metastases. Am J SurgPathol. 2000; 24:197-202.

33. Ishikawa Y, Horiguchi J, Toya $\mathrm{H}$, et al. Triple-negative breast cancer: histological subtypes and immunohsitochemical and clinicopathological features. Cancer Sci. 2011;102(3):656-62.

34. Montagna E, Maisonneuve P, Rotmensz N, et al. Heterogeneity of triple negative breast cancer: histologic subtyping to inform the outcome. Clin Breast Cancer. 2013;13(1):31-9.

35. Monhollen L, Morrison C, Ademuyiwa FO, et al. Pleomorphic lobular carcinoma: a distinctive clinical and molecular breast cancer type. Histopathology. 2012;61(3):365-77.

36. Foulkes WD, Brunet JS, Stefansson IM, et al. The prognostic implication of the basal-like (cyclin E high/p27low/p53+/glomeruloid-microvascul ar proliferation + ) phenotype of BRCA1-related breast cancer. Cancer Res. 2004; 64:830-5.

37. Tian XS, Cong MH, Zhou WH, et al. Clinicopathologic and prognostic characteristics of triple-negative breast cancer. Onkologie. 2008;31(11):610-4.

38. Dogan L, Atalay C, Yilmaz KB, et al. Prognosis in hormone receptor negative breast cancer patients according to ERBB2 status. Neoplasma. 2008;55(6):544-8.

39. El Saghir NS, Seoud M, Khalil MK, et al. Effects of young age at presentation on survival in breast cancer. BMC Cancer. 2006; 6:194.

40. Pogoda K, Niwinska A, Muawska M, et al. Analysis of pattern, time, and risk factors influencing recurrence in triple-negative breast cancer patients. Med Oncol. 2013; 30:388.

41. Stark A, Kapke A, Schultz D, et al. Advanced stages and poorly differentiated grade are associated with an increased risk of HER2/neu positive breast cancer only in white women: findings from a prospective cohort study of African-American and white-American women. Breast Cancer Res Treat. 2008; 107:405-14.

42. Billar JA, Dueck AC, Stucky CC, et al. Triple-negative breast cancers: unique clinical presentations and outcomes. Ann Surg Oncol. 2010;17(Suppl. 3):384-90.

43. Nishimura R, Arima N. Is triple negative a prognostic factor in breast cancer? Breast Cancer. 2008;15(4):303-8.

44. Chivukula M, Striebel JM, Ersahin C, et al. Evaluation of morphologic features to identify "basal-like phenotype" on core needle biopsies of breast. Appl Immunohistochem Mol Morphol 2008; 16:411-6. 
45. Amar S, McCullough AE, Tan W, et al. Prognosis and outcome of small $(<=1 \mathrm{~cm})$, node-negative breast cancer on the basis of hormonal and HER-2 status. Oncologist. 2010;15(10):1043-9.

46. Carey LA, Dees EC, Sawyer L, et al. The triple negative paradox: primary tumor chemosensitivity of breast cancer subtypes. Clin Cancer Res. 2007; 13:2329-34.

47. Wang S, Yang H, Tong F, et al. Response to neoadjuvant therapy and disease free survival in patients with triple-negative breast cancer. GanTo Kagaku Ryoho. 2009;36(2):255-8.

48. Lund MJ, Butler EN, Bumpers HL, et al. High prevalence of triple-negative tumors in an urban cancer center. Cancer. 2008;113(3):608-15.

49. Dent R, Hanna WM, Trudeau M, et al. Pattern of metastatic spread in triple negative breast cancer. Breast Cancer Research Treatment. 2009;115(2):423-8.

50. Haffty BG, Yang Q, Reiss M, et al. Locoregional relapse and distant metastasis in conservatively managed triple negative early-stage breast cancer. J Clin Oncol. 2006;24(36):5652-7.

51. Mayer IA, Abramson VG, Lehmann BD, et al. New Strategies for triple-negative breast cancer-deciphering the heterogeneity. Clin Cancer Res. 2014;20(4):782-90.

52. Lehmann BD, Pietenpol JA, Tan AR. Triple-negative breast cancer: molecular subtypes and new targets for therapy. Am SocClin Oncol Educ Book. 2015:e31-9.

53. Tentori L, Graziani G. Chemopotentiationby PARP inhibitors in cancer therapy. Pharmacol Res. 2005; 52:25.
54. Tutt A, Robson M, Garber JE, et al. Oral poly (ADP-ribose) polymerase inhibitor olaparib in patients with BRCA1 or BRCA2 mutations and advanced breast cancer: a proof-of-concept trial. Lancet. 2010; 376:235.

55. O'Shaughnessy J, Osborne C, Pippen JE, et al. Iniparib plus chemotherapy in metastatic triple-negative breast cancer. N Engl J Med. 2011;364(3):205-14.

56. Isakoff SJ, Overmoyer B, Tung NM, et al. A phase II trial of the PARP inhibitor veliparib (ABT888) and temozolomide for metastatic breast cancer. J Clin Oncol. 2010;28(15s): p. abstr 1019.

57. Tutt A, Robson M, Garber JE, et al. Phase II trial of the oral PARP inhibitor olaparib in BRCA-deficient advanced breast cancer. J Clin Oncol. 2009; 27(18s):p. Abstract CRA501.

58. Gelmon KA, Hirte HW, Robidoux KS, et al. Can we define tumors that will respond to PARP inhibitors? A phase II correlative study of olaparib in advanced serous ovarian cancer and triple-negative breast cancer. J Clin Oncol. 2010;28(15s): p. abstr 3002.

59. Baselga J, Gomez P, Greil R, et al. Randomized phase II study of the anti-epidermal growth factor receptor monoclonal antibody cetuximab with cisplatin versus cisplatin alone in patients with metastatic triple-negative breast cancer. J Clin Oncol. 2013;31(20):2586-92.

60. O'Shaughnessy J, Weckstein D, Vukelja S: Pre-liminary Preliminary results of a randomized phase II study of weekly irinotecan/carboplatin with or without cetuximab in pa-tients patients with metastatic breast cancer. Breast Cancer Res Treat. 2007; 106:S32-S33 (suppl 1; abstr 308). 